\title{
Urinary Tract is a Pandora's Box- Rare, Interesting Finds on Cystoscopy
}

\author{
Dr. Narendra Pai ${ }^{1}$, Dr. Suraj Hegde ${ }^{2}$, Dr. Amulya Cherukumudi ${ }^{3 *}$, Dr. Rajeev T $\mathbf{P}^{4}$ \\ ${ }^{1,2}$ Assistant Professor, Department of Urology, K. S.Hegde Medical Academy, Deralakatte, Mangalore \\ ${ }^{3}$ Junior Resident, Department of General Surgery, K.S.Hedge Medical Academy, Deralakatte, Mangalore \\ ${ }^{4}$ Professor and Head, Department of Urology, K.S.Hedge Medical Academy, Deralakatte, Mangalore
}

*Corresponding Author: Dr. Amulya Cherukumudi, Junior Resident, Department of General Surgery, K.S.Hedge Medical Academy, Deralakatte, Mangalore, E-mail: amulyac1@yahoo.com

\begin{abstract}
In daily practice, we encounter many patients with calculi in various parts of the urinary tract. However, it is rarely a peculiar foreign body such as clips, pins, electric wires, coins etc., which masquerades as a calculus (1). Such patients often have associated psychiatric illnesses or sexual illnesses with autoeroticism which compels them to insert such foreign objects into the urinary tract (2). These can be extracted via endoscopic approach usually, but seldom open approach may be required. Here we present threeinteresting case of foreign bodies within the urinary tract and how we approached each case.
\end{abstract}

Keywords: Foreign body, urinary tract, cystostomy

\section{CASE 1}

Here we discuss about a 34 year old male patient who presented to our Urology outpatient department with complaints of bothersome Lower urinary tract symptoms of intermittency, hesitancy, straining and urgency for duration of 2 months. He, however, had no associated flank pain, fever, haematuria, lithuria or pyuria.

He hasn't undergone any urological interveneetio ns in the past for the same complaints or otherwise.

Patient gives history of an underlying psychiatric disorder and he claims to take his medications irregularly for the same.

On further clinical evaluation, abdominal and rectal examination was normal. On inspection, the external genitalia were appropriate in development for the patient.

Firstly, laboratory investigations were done to identify the cause; urine routine revealed numerous pus cells, which was suggestive of urinary tract infections.

Urine culture and sensitivity was ordered, which demonstrated the growth of Escherichia coli. Ultrasound abdomen and pelvis was ordered, which showed a vesical calculus measuring approximatively $4 \mathrm{~cm}$.
The same was confirmed with an X-ray of the Kidney and Urinary bladder, which revealed a radio-opaque shadow in the urinary bladder with patchy areas of radiolucency.

After the initial work-up, patient was posted for aurethrocystoscopy.

It was found that the urethra, bladder neck and visible portion of the prostate gland were normal.

In the bladder, a foreign body was noted measuring approximately $5 \mathrm{~cm}$.

Bilateral ureteric orifice was identified and deemed to be normal, with no evidence of bladder trabeculations or growth.

As it was a large calculus to clear endoscopically, we decided to proceed with open suprapubic cystolithotomy.

On inspection, a yellowish-white, irregular and hard structure was noted, free from the bladder mucosa, and was extracted in toto. To our surprise, we discovered that it was an electric wire of $65 \mathrm{cms}$ length coiled on itself with encrustration.

Post-operative period was uneventful. Patient came for serial follow-up at 2 weeks and 3 months, and had no lower urinary tract symptoms, and is on regular anti-psychotic treatment. 


\section{CASE 2}

Here we describe the presenting complaints of a 65 year old male, who had history of left hip pain since 3 weeks, dull aching, with no restriction of left hip movements. Patient had no preceding history of trauma, any fever or deformity of the hip joint. For these complaints, he sought an orthopedic consultation. X-ray of the pelvis with bilateral hip was done, which revealed a foreign body in the region of urethra. Hence, was referred to the Urology department. On further questioning, he revealed history of insertion of pins through the external urethral orificice, following which he developed obstructive lower urinary tract symptoms. Patient had no known history of psychiatric illness or other associated co-morbidities.

On examination, per-abdomen and per-rectum findings were well within normal limits. On examination of the external genetalia, a foreign body was palpable in the ventral aspect of the penis at the region of the proximal penile urethra.

Patient underwent anurethrocystoscopy, which unveiled the inserted pins within the proximal penile urethra entending to the peno-bulbar urethra junction; the pins were extracted endoscopically. Patient made an uneventful post-operative recovery.

\section{CASE 3}

A 70 year old male presented to the emergency department with history of haematuria with passage of clots since 3 weeks. He also had associated complaints of lower urinary tract symptoms, which were mainly obstructive in nature. Ultrasound abdomen and pelvis revealed a large clot within the urinary bladder measuring $5.5 \times 6 \mathrm{~cm}$.

On further enquiry, patient revealed that he had undergone an endoscopic procedure of the urinary tract, the details of which were unavailable to us at the time of presentation.

Urinary catheterization was attempted, and failed. Hence, patient was posted for an emergency cystoscopy, which was suggestive of a stricture in the bulbar urethra and was dealt with an internal urethrotomy.

Once scope was negotiable beyond the zone of the above mentioned stricture, we found a single vesical calculus with multiple broomsticks in the bladder lumen. These along with the vesical calculus were extracted endoscopically along with several clots. Patient made an uneventful post-operative recovery and is on regular follow-up since.

On retrospective evaluation, patient admitted to have voluntarily inserted the broom sticks to relieve his obstruction, owing to his lower socio-economic status.

\section{Discussion}

Urethra is an unusual route for insertion of foreign objects, which has the tendency to migrate to the proximal urethra or bladder (3).

This migration occurs as a result of manipulation to extract the foreign body or involuntarily due to the intrinsic contraction of the perineal muscles, which can cause injuries to the mucosa of the urethra (4).

Migration without mucosal erosions or trauma is frequently encountered in such patients; the pathogenesis of the same is unknown (1). Once the foreign body migrates to the proximal urethra or bladder, extraction becomes challenging, often requiring endoscopic or open intervention.

There could be various reasons for the insertion of foreign bodies into the lower urinary tract; iatrogenic, psychologic, secondary to trauma or migration from surrounding structures.

Various psychological alterations include sexual curiosity, mental illness and auto-eroticism etc., which compels these patients to insert foreign bodies. Patients have also been found to insert additional foreign bodies in an attempt to extract the previously lodged items $(3,4)$.

Self-insertion of a foreign body via the urethra is unusual and uncommon, hence is often missed during history taking and clinical examination.

In our first case, patient was questioned retrospectively after the surgery, which confirmed the history of self-insertion of the wire per-urethra.

Even when history fails to give us the relevant information, a basic radiologic examination such as pelvic X-ray can reveal the location, size and number of the foreign bodies (2).Ultrasound of the pelvis and computed tomography maybe necessary to confirm the diagnosis, and to establish a sound treatment plan for such patients.

Treatment for foreign bodies is almost always endoscopic to avoid urethral and bladder trauma, and in majority of the cases is sufficient to extract the foreign body.In our second case, we were able to extract the pins from the proximal urethra.

However, in case of difficult extraction, open procedures are resorted to, such as in our first 
case, where extraction was done via open suprapubic cystostomy $(2,3)$.

\section{CONCLUSION}

foreign bodies in the urinary tract are an unusual and uncommon finding in routine clinical practice, and hence one must suspect it in case of vague histories and associated psychiatric illnesses.

\section{REFERENCES}

[1] Ku JH, Lee CS, Jeon YS, Kim ME, Lee NK. A Foreign body in the urethra: a case report. Ko rean J Urol. 1997; 38:219-221.
[2] Johnin K, Kushima M, Koizumi S, Okada Y. Percutaneous transvesical retrieval of foreign bodies penetrating the urethra. $\mathrm{J}$

Urol. 1999;161:915-916

[3] Cho DS, Kim SJ, Choi JB. Foreign bodies in urethra and bladder by implements used during sex behavior. Korean J Urol. 2003;44:11311134

[4] Rahman NU, Elliott SP, McAninch JW. Selfinflicted male urethral foreign body insertion: endoscopic management and complications. BJU Int. 2004;94:1051-1053.

Citation: Dr. Narendra Pai, et.al. Urinary Tract is a Pandora's Box-Rare, Interesting Finds on Cystoscopy. ARC Journal of Urology.2019; 4(2): 11-14.doi:dx.doi.org/10.20431/2456-060X.0402003.

Copyright: (C) 2019 Authors. This is an open-access article distributed under the terms of the Creative Commons Attribution License, which permits unrestricted use, distribution, and reproduction in any medium, provided the original author and source are credited. 